\title{
A minimax inequality and its applications to fixed point theorems in CAT(0) spaces
}

\author{
S Shabanian and SM Vaezpour ${ }^{*}$
}

* Correspondence: vaez@aut.ac.ir Department of Mathematics and Computer Science, Amirkabir University of Technology, Hafez Ave., P.O. Box 15875-4413, Tehran, Iran

\author{
Abstract \\ In this paper, a CAT(0) version of famous Fan's minimax inequality is established and \\ as its application, we obtain some fixed point theorems and best approximation \\ theorems in CAT(0) spaces. \\ 2000 Mathematics Subject Classification: 47H10. \\ Keywords: CAT(0) space, minimax inequality, fixed point
}

\section{Introduction}

A metric space is a CAT(0) space if it is geodesically connected and if every geodesic triangle in this space is at least as thin as its comparison triangle in Euclidean plane. $\mathrm{CAT}(0)$ spaces play fundamental role in various areas of mathematics [1]. Moreover, there are applications in biology and computer science as well $[2,3]$.

Fixed point theory in a CAT(0) space was first studied by Kirk [4]. Since then, the fixed point theory for single valued and multivalued mappings in $\mathrm{CAT}(0)$ spaces has been developed [5-8].

The famous Knaster-Kuratowski-Mazurkiewicz theorem (in short, KKM theorem) and its generalization have a fundamental importance in modern nonlinear analysis $[9,10]$. Recently, Niculescu and Roventa established the KKM mapping principle for CAT(0) spaces [11].

In this paper, a minimax inequality in $\mathrm{CAT}(0)$ spaces is established and as its application, some fixed point and best approximation theorems in CAT(0) spaces are proved.

\section{Preliminaries}

Let $(X, d)$ be a metric space. A geodesic path joining $x \in X$ to $y \in Y$ (briefly, a geodesic from $x$ to $y$ ) is a map $c$ from a closed interval $[0, l] \subseteq \mathbb{R}$ to $X$ such that $c(0)=x, c$ $(l)=y$ and $d\left(c(t), c\left(t^{\prime}\right)\right)=\left|t-t^{\prime}\right|$ for all $t, t^{\prime} \in[0, l]$. In particular, $c$ is an isometry and $d(x, y)=l$. The image of $c$ is called a geodesic segment joining $x$ and $y$. When it is unique, this geodesic is denoted by $[x, y]$.

The metric space $(X, d)$ is said to be a geodesic space if every two points of $X$ are joined by a geodesic, and $X$ is said to be uniquely geodesic if there is exactly one geodesic joining $x$ and $y$ for each $x, y \in X$. A subset $Y$ of $X$ is said to be convex if $Y$ includes every geodesic segment joining any of two its points.

(c) 2011 Shabanian and Vaezpour; licensee Springer. This is an Open Access article distributed under the terms of the Creative Commons Attribution License (http://creativecommons.org/licenses/by/2.0), which permits unrestricted use, distribution, and reproduction in any medium, provided the original work is properly cited. 
A geodesic triangle $\Delta\left(x_{1}, x_{2}, x_{3}\right)$ in a geodesic space consists of three points $x_{1}, x_{2}, x_{3}$ in $X$ (the vertices of $\Delta$ ) and a geodesic segment between each pair of vertices (the edges of $\Delta)$. A comparison triangle for geodesic triangle $\Delta\left(x_{1}, x_{2}, x_{3}\right)$ in $(X, d)$ is a triangle $\bar{\Delta}\left(x_{1}, x_{2}, x_{3}\right):=\Delta\left(\bar{x}_{1}, \bar{x}_{2}, \bar{x}_{3}\right)$ in the Euclidian plane $\mathbb{E}^{2}$ such that

$$
d_{\mathbb{E}^{2}}\left(\bar{x}_{i}, \bar{x}_{j}\right)=d\left(x_{i}, x_{j}\right)
$$

for $i, j \in\{1,2,3\}$.

A geodesic space is called a CAT(0) space if all geodesic triangles satisfy the CAT(0) inequality:

For every geodesic triangle, $\Delta$ in $X$ and its comparison triangle $\bar{\Delta}$ in $\mathbb{E}^{2}$, if $x, y \in \Delta$, and $\bar{x}, \bar{y}$ are comparison points in $\bar{\Delta}$, then

$$
d(x, y) \leq d_{\mathbb{E}^{2}}(\bar{x}, \bar{y}) .
$$

We now collect some elementary facts about CAT(0) spaces which will be used in the proofs of our main results.

Lemma 2.1 [1]Every $C A T(0)$ space $(X, d)$ is uniquely geodesic, and the balls in $(X, d)$ are convex.

Lemma $2.2[12]$ Let $(X, d)$ be a $C A T(0)$ space. Then,

1. for each $x, y \in X$ such that $x \neq y$ then $d(x, z)+d(z, y)=d(x, y)$ if and only if $z \in$ $[x, y]$,

2. for each $x, y \in X$ and $t \in[0,1]$, there exists a unique point $z \in[x, y]$ such that $d$ $(x, z)=t d(x, y)$ and $d(y, z)=(1-t) d(x, y)$.

Recall that we say a topological space $K$ has the fixed point property if every continuous map $f: K \rightarrow K$ has a fixed point. Let $X$ and $Y$ be topological Hausdorff spaces, $B$ $\subseteq Y$ and $T: X \rightarrow Y$ be a multivalued map with nonempty values. Define

$$
T^{-}(B)=\{x \in X: T(x) \cap B \neq \emptyset\},
$$

and let $\operatorname{int}(B), \partial B$ and $\mathcal{F}(B)$ denote the interior, boundary and the set of all nonempty finite subsets of $B$.

Let $E$ be a $C A T(0)$ space and $F \subseteq E$. Recall that the notion of a convex hull is introduced via the formula

$$
\operatorname{co}(F)=\bigcup_{n=0}^{\infty} F_{n}
$$

where $F_{0}=F$ and for $n \geq 1$, the set $F_{n}$ consists of all points in $E$ which lie on geodesics which start and end in $F_{n-1}$. The convex hull of a finite subset is not necessarily closed, but in any locally convex Hausdorff space, if $K_{1}, \ldots, K_{n}$ are compact convex subsets, then the convex hull of their union is compact too [13].

Definition 2.1 [11] Let $C$ be a nonempty subset of a CAT(0) space $E$. A multivalued mapping $G: C \rightarrow 2^{E}$ is said a KKM mapping if

$$
\operatorname{co}(F) \subseteq \bigcup_{x \in F} G(x)
$$

for every nonempty finite set $F \in \mathcal{F}(C)$.

Example 2.1 Let $C$ be a convex subset of CAT(0) space $E$ and $f: C \rightarrow \mathbb{R}$ be such that for each $x_{1}, \ldots, x_{n} \in X$ if $x \in \operatorname{co}\left(\left\{x_{1}, \ldots, x_{n}\right\}\right)$, then there exist $a_{1}, \ldots, a_{n} \in \mathbb{R}$ with 


$$
\begin{aligned}
\phi(x)= & \{y \in C: f(y) \leq f(x)\} . \text { such that } f(x) \leq \sum_{i=1}^{n} a_{i} f\left(x_{i}\right) . \text { For each } x \in C \text { define } \\
& \phi(x)=\{y \in C: f(y) \leq f(x)\} .
\end{aligned}
$$

We show that $\varphi$ is a KKM map. By contradiction, suppose $y \in \operatorname{co}\left(\left\{x_{1}, \ldots, x_{n}\right\}\right)$ and $y \notin$ $\mathrm{U}_{i} \varphi\left(x_{i}\right)$. Therefore, there exist $a_{1}, \ldots, a_{n} \in \mathbb{R}$ with $\sum_{i=1}^{n} a_{i}=1$ such that $f(y) \leq \sum_{i=1}^{n} a_{i} f\left(x_{i}\right)$. Since $f\left(x_{i}\right)<f(y)$ for each $i=1, \ldots, n$, so we have a contradiction. Thus, $\varphi$ is a KKM map.

Definition 2.2 We say that a CAT(0) space $X$ has the convex hull finite property if the closed convex hull of every nonempty finite family of points of $X$ has the fixed point property.

Example 2.2 [14] In a locally compact CAT(0) space, the closed convex hull of each finite family of points has the fixed point property. So, every locally compact CAT(0) space has the convex hull finite property.

The following important result is established in [11].

Theorem 2.1 (KKM mapping principle) Suppose that E is a complete CAT(0) space with the convex hull finite property and $X$ is a nonempty subset of E. Furthermore, suppose $M: X \rightarrow 2^{X}$ is a KKM mapping with closed values. Then, if $M(z)$ is compact for some $z \in X$, then

$$
\bigcap_{x \in X} M(x) \neq \emptyset .
$$

\section{Main results}

The following theorem is a direct application of KKM mapping principle.

Theorem 3.1 Suppose $X$ is a compact subset of a complete CAT(O) space $E$ with convex hull finite property and $F: X \rightarrow E$ is continuous. Then, there exists $y_{0} \in X$ such that

$$
d\left(y_{0}, F\left(y_{0}\right)\right)=\inf _{x \in X} d\left(x, F\left(y_{0}\right)\right) .
$$

Proof. Consider the map $G: X \rightarrow 2^{E}$ defined by

$$
G(x)=\{y \in X: d(y, F(y)) \leq d(x, F(y))\} .
$$

Since $F$ is continuous, so $G(x)$ is closed for every $x \in X$. We claim that

$$
\operatorname{co}(A) \subseteq \bigcup_{x \in A} G(x),
$$

for all finite set $A \subseteq X$. On the contrary, there exists $\left\{x_{1}, \ldots, x_{n}\right\} \subseteq X$ and $y \in \operatorname{co}\left(\left\{x_{1}, \ldots\right.\right.$, $\left.\left.x_{n}\right\}\right)$ such that $y \notin \bigcup_{i} G\left(x_{i}\right)$. This clearly implies

$$
d\left(x_{i}, F(y)\right)<d(y, F(y)),
$$

for $i=1, \ldots, n$. Hence, $x_{i} \in B(F(y), d(y, F(y)))$ for $i=1, \ldots, n$. Therefore, we have

$$
c o\left(\left\{x_{1}, \ldots, x_{n}\right\}\right) \subseteq B(F(y), d(y, F(y))),
$$

which implies that $y \in B(F(y), d(y, F(y)))$. Clearly, this gets a contradiction.

By compactness of $X$, we deduce that $G(x)$ is compact for every $x \in X$. Therefore, there exists $y_{0} \in \cap_{x \in X} G(x)$. This clearly implies $d\left(y_{0}, F\left(y_{0}\right)\right) \leq d\left(x, F\left(y_{0}\right)\right)$ for every $x \in$ $X$ which implies 


$$
d\left(y_{0}, F\left(y_{0}\right)\right)=\inf _{x \in X} d\left(x, F\left(y_{0}\right)\right)
$$

and the proof is complete. $\square$

Theorem 3.2 Suppose $X$ is a compact subset of a complete CAT(O) space $E$ with convex hull finite property and $F: X \rightarrow E$ is a continuous map such that for every $c \in X$, with $c \neq F(c)$, there exists $\alpha \in(0,1)$ such that

$$
X \cap B(F(c),(1-\alpha) d(c, F(c))) \neq \emptyset .
$$

Then, $F$ has a fixed point.

Proof. By Theorem 3.1, there exists $y_{0} \in X$ such that

$$
d\left(y_{0}, F\left(y_{0}\right)\right)=\inf _{x \in X} d\left(x, F\left(y_{0}\right)\right) .
$$

We claim that $y_{0}$ is a fixed point of $F$. Indeed, assume not, i.e., $y_{0} \neq F\left(y_{0}\right)$. Then, our assumption on $X$ implies the existence of $\alpha \in(0,1)$ such that

$$
X \cap B\left(F\left(y_{0}\right),(1-\alpha) d\left(y_{0}, F\left(y_{0}\right)\right)\right) \neq \emptyset .
$$

Let $x \in X \cap B\left(F\left(y_{0}\right),(1-\alpha) d\left(y_{0}, F\left(y_{0}\right)\right)\right)$. Clearly, $x \neq y_{0}$, and we have

$$
d\left(x, F\left(y_{0}\right)\right)<(1-\alpha) d\left(y_{0}, F\left(y_{0}\right)\right) .
$$

Since $d\left(y_{0}, F\left(y_{0}\right)\right) \leq d\left(x, F\left(y_{0}\right)\right)$, we clearly get a contradiction and this completes the proof. $\square$

Definition 3.1 Let $E$ be a $C A T(0)$ space, and $C$ be a convex subset of $E$. A function $f$ $: C \rightarrow \mathbb{R}$ is said to be metrically quasi-concave (resp., metrically quasi-convex) if for each $\lambda \in \mathbb{R}$, the set $\{x \in C: f(x)>\lambda\}$ (resp., $\{x \rightarrow C: f(x)<\lambda\}$ ) is convex.

Example 3.1 Consider Hilbert space $\lambda^{2}$ consisting of all complex sequences with the norm $\|x\|=\left(\sum_{i=1}^{\infty} \xi_{i}^{2}\right)^{\frac{1}{2}}$, where $x=\left(\xi_{j}\right) \in \lambda^{2}$. Define the functions $f, g: \lambda^{2} \rightarrow \mathbb{R}$ defined by

$$
f(x)=\left\{\begin{array}{cc}
0 & x=0 \\
\frac{1}{\|x\|} & x \neq 0
\end{array}\right.
$$

and $g(x)=\|x\|$. It is easy to see that $f$ is metrically quasi-concave and is not quasiconvex, and $g$ is metrically quasi-convex and not metrically quasi-concave.

Lemma 3.1 Let $C$ be a convex subset of a $C A T(0)$ space $X$, and the function $f: C \times C$ $\rightarrow \mathbb{R}$ satisfies the following conditions.

1. for each $x \in C$, the function $f(\cdot, x): C \rightarrow \mathbb{R}$ is metrically quasi-concave (resp., metrically quasi-convex),

2. there exists $\gamma \in \mathbb{R}$ such that $f(x, x) \leq \gamma($ resp., $f(x, x) \geq)$ for each $x \in C$.

Then, the mapping $G: C \rightarrow 2^{X}$, which is defined by

$$
G(x)=\{y \in C: f(x, y) \leq \gamma\} \text { (resp., } G(x)=\{y \in C: f(x, y) \geq \gamma\}),
$$

is a KKM mapping.

Proof. The conclusion is proved for the concave case, the convex case is completely similar. On the contrary assume that $G$ is not a KKM mapping. Suppose that there exists a finite subset $A=\left\{x_{1}, \ldots, x_{n}\right\}$ of $C$ and a point $x_{0} \in \operatorname{co}(A)$ such that $x_{0} \notin G\left(x_{i}\right)$ for 
each $i=1, \ldots, n$. By setting

$$
\lambda=\min \left\{f\left(x_{i}, x_{0}\right): i=1, \ldots, n\right\}>\gamma,
$$

and

$$
B=\left\{z \in C: f\left(z, x_{0}\right)>\lambda_{0}\right\},
$$

where $\lambda>\lambda_{0}>\gamma$. For each $i$, we have $x_{i} \in B$. According to hypothesis $1, B$ is convex and hence $c o(A) \subseteq B$. So, $x_{0} \in B$, and we have $f\left(x_{0}, x_{0}\right)>\lambda_{0}>\gamma$ which is a contradiction by (2). Thus, $G$ is a KKM mapping. $\square$

Definition 3.2 Let $X, Y$ be CAT(0) spaces. A map $F: X \rightarrow 2^{Y}$ is said to be

- upper semicontinuous if for each closed set $B \subseteq Y, F^{-}(B)$ is closed in $X$.

- lower semicontinuous if for each open set $B \subseteq Y, F(B)$ is open in $X$.

It is well known that if $F(x)$ is compact for each $x \in X$, then $F$ is upper semicontinuous if and only if for each $x \in X$ and $\varepsilon>0$, there exist $\Delta>0$ such that for each $x^{\prime} \in B$ $(x, \Delta)$, we have $F\left(x^{\prime}\right) \subseteq B(F(x), \varepsilon)$.

The following is a $\operatorname{CAT}(0)$ version of the Fan's minimax inequality [15].

Theorem 3.3 Suppose $C$ is a compact and convex subset of a complete CAT(0) space $E$ with convex hull finite property and $f: C \times C \rightarrow \mathbb{R}$ satisfies the following,

1. for each $x \in C$, the function $f(x, \cdot): C \rightarrow \mathbb{R}$ is lower semicontinuous (resp., upper semicontinuous),

2. for each $y \in C$, the function $f(\cdot, y): C \rightarrow \mathbb{R}$ is metrically quasi-concave (resp., metrically quasi-convex),

3. there exists $\gamma \in \mathbb{R}$ such that $f(x, x) \leq \gamma($ resp., $f(x, x) \geq \gamma)$ for each $x \in C$.

Then, there exists a $y_{0} \in C$ such that $f\left(x, y_{0}\right) \leq \gamma$ (resp., $f\left(x, y_{0}\right) \geq \gamma$ ) for all $x \in C$ and hence

$$
\begin{gathered}
\sup _{x \in C} f\left(x, y_{0}\right) \leq \sup _{x \in C} f(x, x) \\
\text { (resp., } \left.\inf _{x \in C} f\left(x, y_{0}\right) \geq \inf _{x \in C} f(x, x)\right) .
\end{gathered}
$$

Proof. By hypothesis $3, \lambda=\sup _{x \in C} f(x, x)<\infty$. For each $x \in C$, define the mapping $G$ $: C \rightarrow 2^{C}$ by

$$
G(x)=\{y \in C: f(x, y) \leq \lambda\},
$$

which is closed by hypothesis (1). By Lemma 3.1, G is a KKM mapping. By Theorem 2.1 ,

$$
\bigcap_{x \in C} G(x) \neq \emptyset
$$

Therefore, there exists a $y_{0} \in \bigcap_{x \in C} G(x)$. Thus, $f\left(x, y_{0}\right) \leq \lambda$ for every $x \in C$. Hence,

$$
\sup _{x \in C} f\left(x, y_{0}\right) \leq \sup _{x \in C} f(x, x) .
$$


This completes the proof. $\square$

Definition 3.3 Let $X$ be a CAT(0) space and $D \subseteq X$. The map $G: D \rightarrow 2^{X}$ is called quasi-convex if the set $G^{-}(C)$ is convex for each convex subset $C$ of $Y$.

Theorem 3.4 Suppose $X$ is a compact subset of a complete CAT(O) space $E$ with convex hull finite property and $F, G: X \rightarrow 2^{E}$ are upper semicontinuous maps with nonempty compact convex values and $G$ is quasi-convex. Then, there exists $x_{0} \in X$ such that

$$
d\left(G\left(x_{0}\right), F\left(x_{0}\right)\right)=\inf _{x \in X} d\left(G(x), F\left(x_{0}\right)\right) .
$$

Proof. Let $H: X \rightarrow 2^{X}$ be defined by

$$
H(y)=\{x \in X: d(G(x), F(x)) \leq d(G(y), F(x))\} .
$$

For each $y \in X$, since $y \in H(y)$, so $H(y) \neq \varnothing$.

We claim that $H(y)$ is closed for each $y \in X$. Suppose that $\left\{y_{n}\right\}$ be a sequence in $H(y)$ such that $y_{n} \rightarrow y^{*}$. We show that $y^{*} \in H(y)$. Let $\varepsilon>0$ be arbitrary. Since $F$ is upper semicontinuous with compact values, so there exists $N_{1}$ such that for each $n \geq N_{1}$, we have

$$
F\left(y_{n}\right) \subseteq \bar{B}\left(F\left(\gamma^{*}\right), \varepsilon\right) .
$$

Similarly, we can prove there exists $N_{1}$ such that for each $n \geq N_{2}$, we have

$$
G\left(y_{n}\right) \subseteq \bar{B}\left(G\left(\gamma^{*}\right), \varepsilon\right) .
$$

Let $N=\max \left\{N_{1}, N_{2}\right\}$. Then, we have

$$
\begin{aligned}
d\left(G\left(y^{*}\right), F\left(y^{*}\right)\right) & \leq d\left(G\left(y^{*}\right), G\left(y_{n}\right)\right)+d\left(G\left(y_{n}\right), F\left(y_{n}\right)\right)+d\left(F\left(y_{n}\right), F\left(y^{*}\right)\right) \\
& \leq 2 \varepsilon+d\left(G\left(y_{n}\right), F\left(y_{n}\right)\right) \\
& \leq 2 \varepsilon+d\left(G(y), F\left(y_{n}\right)\right) \\
& \leq 2 \varepsilon+d\left(G(y), F\left(y^{*}\right)\right)+d\left(F\left(y^{*}\right), F\left(y_{n}\right)\right) \\
& \leq 3 \varepsilon+d\left(G(\gamma), F\left(y^{*}\right)\right) .
\end{aligned}
$$

Since $\varepsilon$ was arbitrary, so

$$
d\left(G\left(\gamma^{*}\right), F\left(\gamma^{*}\right)\right) \leq d\left(G(\gamma), F\left(\gamma^{*}\right)\right),
$$

and this proves our claim.

Now, we show that for each $A \in \mathcal{F}(X), c o(A) \subseteq H(A)$. On the contrary, suppose $c o(A)$ $\nsubseteq H(A)$ for some $A \in \mathcal{F}(X)$. Then, there exists $y \in \operatorname{co}(A)$ such that $y \notin H(a)$ for every $a$ $\in A$. Therefore,

$$
d(G(a), F(\gamma))<d(G(\gamma), F(\gamma)),
$$

for some $a \in A$. For each $a \in A$, we have

$$
G(a) \bigcap\left(\bigcup_{\gamma^{\prime} \in F(y)} B\left(y^{\prime}, \max _{b \in A} d(G(a), F(y))\right)\right) \neq \emptyset .
$$

Since $F(y)$ is convex, so

$$
\left.\bigcup_{\gamma^{\prime} \in F(y)} B\left(\gamma^{\prime}, \max _{b \in A} d(G(b), F(y))\right)\right)
$$


is convex. This shows that

$$
G(\gamma) \bigcap\left(\bigcup_{\gamma^{\prime} \in F(y)} B\left(\gamma^{\prime}, \max _{b \in A} d(G(b), F(\gamma))\right)\right) \neq \emptyset,
$$

because $G$ is quasi-convex. Therefore, by (1), we have

$$
d(G(y), F(\gamma)) \leq \max _{b \in A} d(G(b), F(\gamma))<d(G(y), F(y))
$$

This is a contradiction. Now, by Theorem 2.1, it follow that there exists $x_{0} \in X$ such that

$$
x_{0} \in \cap_{y \in X} H(y) .
$$

Hence,

$$
d\left(G\left(x_{0}\right), F\left(x_{0}\right)\right)=\inf _{x \in X} d\left(G(x), F\left(x_{0}\right)\right) .
$$

This completes the proof. $\square$

Corollary 3.1 Suppose $X$ is a compact subset of a complete CAT(O) space E with convex hull finite property and $G: X \rightarrow 2^{X}$ is an onto, quasi-convex and upper semicontinuous map with nonempty compact convex values and $f: X \rightarrow X$ is a continuous single valued map. Then, there exists $x_{0} \in X$ such that $f\left(x_{0}\right) \in G\left(x_{0}\right)$.

Corollary 3.2 Suppose $X$ is a compact subset of a complete CAT(O) space E with convex hull finite property and $G: X \rightarrow 2^{X}$ is a quasi-convex and an upper semicontinuous map with nonempty compact convex values. Then, there exists $x_{0} \in X$ such that

$$
d\left(x_{0}, G\left(x_{0}\right)\right)=\inf _{x \in X} d\left(G(x), x_{0}\right) .
$$

Corollary 3.3 Suppose $X$ is a compact subset of a complete CAT(O) space E with convex hull finite property and $G: X \rightarrow 2^{X}$ is an upper semicontinuous map with nonempty compact convex values. Then, there exists $x_{0} \in X$ such that

$$
d\left(G\left(x_{0}\right), x_{0}\right)=\inf _{x \in X} d\left(G\left(x_{0}\right), x\right) .
$$

Moreover, if $x_{0} \notin G\left(x_{0}\right)$ then $x_{0} \in \partial X$.

Proof. By Theorem 3.4, clearly there exists $x_{0} \in X$ such that

$$
d\left(G\left(x_{0}\right), x_{0}\right)=\inf _{x \in X} d\left(G\left(x_{0}\right), x\right) .
$$

Suppose $x_{0} \notin G\left(x_{0}\right)$. Since $G$ has compact values, so $d\left(x_{0}, G\left(x_{0}\right)\right)=r>0$. We prove that $x_{0} \in \partial X$. Assume, it is not. Then, $x_{0} \in \operatorname{int}(X)$. Therefore, there exists an $\varepsilon \in(0, r)$ such that $B\left(x_{0}, \varepsilon\right) \subseteq X$. Take $z_{0} \in G\left(x_{0}\right)$ such that $d\left(x_{0}, z_{0}\right)<r+\frac{\varepsilon}{2}$. By Lemma 2.2(2), there exists $y_{0} \in\left[x_{0}, z_{0}\right]$ such that $d\left(x_{0}, y_{0}\right)=\frac{\varepsilon}{2}$. Again by Lemma 2.2(1), we have

$$
d\left(y_{0}, G\left(x_{0}\right)\right) \leq d\left(y_{0}, z_{0}\right)=d\left(x_{0}, z_{0}\right)-d\left(x_{0}, y_{0}\right)<r=d\left(x_{0}, G\left(x_{0}\right),\right.
$$

which is a contradiction by (2). Therefore, $x_{0} \in \partial X$. $\square$

Corollary 3.4 Suppose $X$ is a compact subset of a complete CAT(O) space E with convex hull finite property and $G: X \rightarrow 2^{X}$ is an upper semicontinuous map with nonempty compact convex values. If $G(x) \cap X=\varnothing$ for all $x \in \partial X$, then $G$ has a fixed point. 
Proof. On the contrary, assume that $G$ does not have a fixed point. Therefore, by Theorem 3.4, there exists $x_{0} \in \partial X$ such that

$$
0<d\left(x_{0}, G x_{0}\right) \leq d\left(x, G x_{0}\right),
$$

for all $x \in X$. Since $x_{0} \in \partial X$, we have $G x_{0} \cap X \neq \varnothing$. This is a contradiction by (3).

If in Theorem 3.4, $G$ is single valued, then it reduces to the following analog of Fan's best approximation to single-valued mappings in $\operatorname{CAT}(0)$ spaces.

Corollary 3.5 Suppose $X$ is a compact subset of $E$ and $G: X \rightarrow E$ is a continuous map. Then, there exists $x_{0} \in X$ such that

$$
d\left(G x_{0}, x_{0}\right) \leq d\left(G x_{0}, x\right),
$$

for all $x \in X$.

The following is an analog of Fan's fixed point theorem in CAT(0) spaces [16].

Theorem 3.5 Suppose $X$ is a compact subset of a complete CAT(O) space $E$ with convex hull finite property and $G: X \rightarrow E$ is a continuous map and for every $x \in X$ with $x$ $\neq G x$,

$$
(x, G x]=[x, G x] \backslash\{x\},
$$

contains at least one point of $X$, then $G$ has a fixed point.

Proof. By the Corollary 3.5, there exists $x_{0} \in X$ such that

$$
d\left(x_{0}, G x_{0}\right) \leq d\left(x, G x_{0}\right),
$$

for all $x \in X$.

We claim that $x_{0}$ is a fixed point of $T$. On the contrary, assume that $x_{0} \neq G x_{0}$. Then, by assumptions, there exists $z \in X$ such that $z \in\left(x_{0}, G x_{0}\right]$.

Therefore,

$$
d\left(z, G x_{0}\right)=d\left(x_{0}, G x_{0}\right)-d\left(x_{0}, z\right)<d\left(x_{0}, G x_{0}\right),
$$

which by (4) it is a contradiction. $\square$

Authors' contributions

All authors read and approved the final manuscript.

\section{Competing interests}

The authors declare that they have no competing interests.

Received: 25 December 2010 Accepted: 4 October 2011 Published: 4 October 2011

\section{References}

1. Bridson, M, Haefliger, A: Metric Spaces and Non-positive Curvature. Springer, Berlin (1999)

2. Bartolini, I, Ciaccia, P, Patella, M: String matching with metric trees using an approximate distance. In SPIR Lecture Notes in Computer Science, vol. 2476,Springer, Berlin (1999)

3. Semple, C: Phylogenetics. Oxford Lecture Series in Mathematics and Its Application. Oxford University Press, Oxford (2003)

4. Kirk, WA: Geodesic geometry and fixed point theory. In Seminar of Mathematical Analysis, vol. 64, pp. 193-225.Univ Sevilla Secr Publ., Seville (2003)

5. Kaewcharoen, A, Kirk, WA: Proximinality in geodesic spaces. Abstr Appl Anal 2006, 1-10 (2006). Article ID 43591

6. Kirk, WA: Fixed point theorems in CAT(0) spaces and R-trees. Fixed Point Theory Appl. 4, 309-316 (2004)

7. Kirk, WA: Geodesic geometry and fixed point theory II. International Conference on Fixed Point Theory and Applications. pp. 113-142.Yokohama Publisher, Yokohama (2004)

8. Kirk, WA, Payanak, B: A concept of convergence in geodesic spaces. Nonlinear Anal. 68, 3689-3696 (2008). doi:10.1016/j. na.2007.04.011

9. Kirk, WA, Sims, B, Yuan, GX: The Knaster-Kuratowski and Mazurkiewicz theory in hyperconvex metric spaces and some of its applications. Nonlinear Anal. 39, 611-627 (2000). doi:10.1016/S0362-546X(98)00225-9 
10. Knaster, B, Kuratowski, C, Mazurkiewicz, S: Ein bewies des fix-punksatzes fur n-dimensionale simplexe. Fund Math. 14, 132-137 (1929)

11. Niculescu, CP, Roventa, L: Fan's inequality in geodesic spaces. Appl Math Lett. 22, 1529-1533 (2009). doi:10.1016/j. aml.2009.03.020

12. Dhompongsa, S, Panyanak, B: On $\Delta$-convergence theorem in CAT(0) spaces. Comput Math Appl. 56, 2572-2579 (2008). doi:10.1016/j.camwa.2008.05.036

13. Day, MM: Normed Linear Spaces, Ergebnisse Der Mathematik Und Ihrer Grenzgebiete. Springer, New York (1973)

14. Niculescu, CP, Roventa, L: Schauder fixed point theorem in spaces with global nonpositive urvature. Fixed Point Theory Appl (2009). Article ID 906727

15. Fan, K: A minimax inequality and applications. In: Shisha O (ed.) Inequalities, vol. 3, pp. 103-113. Academic Press, New York (1972)

16. Fan, K: Extensions of tow fixed point theorems of F. E Browder Math Z. 112, 234-240 (1969)

doi:10.1186/1687-1812-2011-61

Cite this article as: Shabanian and Vaezpour: A minimax inequality and its applications to fixed point theorems in CAT(0) spaces. Fixed Point Theory and Applications $20112011: 61$.

\section{Submit your manuscript to a SpringerOpen ${ }^{\circ}$} journal and benefit from:

- Convenient online submission

- Rigorous peer review

- Immediate publication on acceptance

- Open access: articles freely available online

- High visibility within the field

- Retaining the copyright to your article

Submit your next manuscript at $\gg$ springeropen.com 